\title{
ON THE GENERATORS OF THE FIRST HOMOLOGY WITH COMPACT SUPPORTS OF THE WEIERSTRASS FAMILY IN CHARACTERISTIC ZERO
}

BY

GORO C. KATO

\begin{abstract}
Let $\mathbf{W}_{\mathbf{Q}}=\operatorname{Proj}\left(\mathbf{Q}\left[g_{2}, g_{3}, X, Y, Z\right] /\right.$ (homogeneous ideal generated by $\left.\left.-Y^{2} Z+4 X^{3}-g_{2} X Z^{2}-g_{3} Z^{3}\right)\right)$. This is said to be the Weierstrass Family over the field $\mathbf{Q}$. Then the first homology with compact supports of the Weierstrass Family is computed explicitly, i.e., it is generated by $\left\{C^{-k} d X \wedge d Y\right\}_{k \geq 1}$ and $\left\{X C^{-k} d X \wedge d Y\right\}_{k>1}$ over the ring $\mathbf{Q}\left[g_{2}, g_{3}\right]$, where $C$ is a polynomial $Y^{2}-4 X^{3}+$ $g_{2} X+g_{3}$. When one tensors the homology of the Weierstrass Family with $\Delta^{-1} \mathbf{Q}\left[g_{2}, g_{3}\right]$, being localized at the discriminant $\Delta=g_{2}^{3}-27 g_{3}^{2}$, over $\mathbf{Q}\left[g_{2}, g_{3}\right]$, the first homology is generated by $C^{-1} d X \wedge d Y$ and $X C^{-1} d X \wedge d Y$. One also obtains the first homologies with compact supports of singular fibres over $\wp=\left(g_{2}\right.$ $\left.=g_{3}=0\right)$ and $\wp=\left(g_{2}=3, g_{3}=1\right)$ as corollaries.
\end{abstract}

Introduction. We wish to compute the $\mathrm{Q}\left[g_{2}, g_{3}\right]$-adic homology with compact supports of the Weierstrass Family $\mathbf{W}_{\mathbf{Q}}$, where

$$
\mathbf{W}_{\mathbf{Q}}=\operatorname{Proj}\left(\frac{\mathbf{Q}\left[g_{2}, g_{3}, X, Y, Z\right]}{\text { homogeneous ideal generated by }-Y^{2} Z+4 X^{3}-g_{2} X Z^{2}-g_{3} Z^{3}}\right) \text {. }
$$

We regard the graded ring $\mathbf{Q}\left[g_{2}, g_{3}, X, Y, Z\right]$ as the graded $\mathbf{Q}\left[g_{2}, g_{3}\right]$-algebra such that $X, Y$ and $Z$ each has degree +1 and all the elements of $\mathbf{Q}\left[g_{2}, g_{3}\right]$ have degree zero. Let $U$ be the open subset of $\mathbf{W}_{\mathbf{Q}}$, "the finite points": $U=\mathbf{W}_{\mathbf{Q}} \cap$ $\mathbf{A}^{2}\left(\operatorname{Spec}\left(\mathbf{Q}\left[g_{2}, g_{3}\right]\right)\right)$. This is the closed subscheme of $\mathbf{A}^{2}\left(\operatorname{Spec}\left(\mathbf{Q}\left[g_{2}, g_{3}\right]\right)\right)$ given by $Y^{2}=4 X^{3}-g_{2} X-g_{3}$. Then we have the long exact sequence of the homology with compact supports, $\cdots \rightarrow H_{h-2}^{c}\left(\{\right.$ points at $\left.\infty\}, \mathbf{Q}\left[g_{2}, g_{3}\right]\right) \rightarrow H_{h}^{c}\left(\mathbf{W}_{\mathbf{Q}}, \mathbf{Q}\left[g_{2}, g_{3}\right]\right) \rightarrow$ $H_{h}^{c}\left(U, \mathbf{Q}\left[g_{2}, g_{3}\right]\right) \rightarrow \cdots$. Since $H_{h}^{c}\left(\{\right.$ points at $\left.\infty\}, \mathbf{Q}\left[g_{2}, g_{3}\right]\right)$ vanishes except at $h=0$, we have

$$
H_{h}^{c}\left(U, \mathbf{Q}\left[g_{2}, g_{3}\right]\right)= \begin{cases}H_{h}^{c}\left(\mathbf{W}_{\mathbf{Q}}, \mathbf{Q}\left[g_{2}, g_{3}\right]\right), & h \neq 2, \\ \mathbf{Q}\left[g_{2}, g_{3}\right], & h=2 .\end{cases}
$$

Therefore the knowledge of $H_{h}^{c}\left(U, \mathbf{Q}\left[g_{2}, g_{3}\right]\right), h \geqslant 0$, determines the homology groups of all the fibres in the family over the various points $\wp \in \operatorname{Spec}\left(\mathbf{Q}\left[g_{2}, g_{3}\right]\right)$, i.e.,

$$
E_{p, q}^{2}=\operatorname{Tor}_{p}^{\mathbf{Q}\left[g_{2}, g_{3}\right]}\left(H_{q}^{c}\left(U, \mathbf{Q}\left[g_{2}, g_{3}\right], \mathbf{K}(\wp)\right)\right)
$$

Received by the editors June 8, 1982. The contents of this paper have been presented to the American Mathematical Society Meeting held at Monterey, California, November 19, 1982.

1980 Mathematics Subject Classification. Primary 14G10, 14F30; Secondary 10B10, 14K07.

Key words and phrases. Lifted $p$-adic homology with compact supports, Weierstrass Family, elliptic curves. 
with the abutment $H_{n}^{c}\left(U_{\wp}, \mathbf{K}(\wp)\right)$, where $\mathbf{K}(\wp)$ is the characteristic zero residue field at $\wp \in \operatorname{Spec}\left(\mathbf{Q}\left[g_{2}, g_{3}\right]\right)$.

Let us consider the unequal characteristic case. Suppose that $\theta$ is a complete discrete valuation ring with the quotient field $K$ and residue class field $k$ and suppose that $\underline{A}$ is an $\theta$-algebra. Let $X$ be a scheme over $A=\left(\underline{A} \otimes_{\mathcal{C}} k\right)_{\text {red }}$. Suppose that $\mathbf{K}(\wp)$ is a finite field at $\wp \in \operatorname{Spec}(A)$ and let $W(\mathbf{K}(\wp))$ be the complete discrete valuation ring and denote the quotient field of $W(\mathbf{K}(\wp))$ by $K_{\wp}=W(\mathbf{K}(\wp)) \otimes_{\mathbf{Z}} \mathbf{Q}$. Then the zeta function of the fibre $X_{\wp}$ at $\wp$ is given by

$$
Z_{X_{\varphi}}(T)=\frac{\prod_{p+q=\text { odd }} P_{p, q}(T)}{\prod_{p+q=\text { even }} P_{p, q}(T)}
$$

where $P_{p, q}(T)$ is the reverse characteristic polynomial of the endomorphism of

$$
E_{p, q}^{2}=\operatorname{Tor}_{p}^{A}{ }^{\dagger} \otimes_{\mathbf{z}} \mathbf{Q}\left(H_{q}^{c}\left(X, \underline{A} \dagger \otimes_{\mathbf{Z}} \mathbf{Q}\right), K_{\wp}\right)
$$

induced by the $p^{r}$ th power map, $p^{r}=\operatorname{card}(\mathbf{K}(\wp))$ (see pp. 448-450, [6]). This homological spectral sequence abuts upon $H_{n}^{c}\left(X_{\wp}, K_{\wp}\right)$. Therefore if one knows the lifted $p$-adic homology with compact supports of $X$ over $A, H_{h}^{c}\left(X, \underline{A} \dagger \otimes_{\mathbf{z}} \mathbf{Q}\right), h \geqslant 0$, and the zeta endomorphisms of these groups, (1) determines the zeta function of every fibre over a finite field in the algebraic family $X$ over the ring $A$. These are the subjects in the forthcoming paper [2].

The main result of the paper is the explicitness of the generation of the first homology with compact supports of the entire Weierstrass Family $\mathbf{W}_{\mathbf{Q}}$ in the characteristic zero (Theorem 1) and its consequences.

Acknowledgement. Professor Saul Lubkin has given me guidance and encouragement through our correspondence and our conversations over the phone. He further suggested to work initially with the case of characteristic zero. I am deeply indebted to Professor Saul Lubkin for his teaching.

1. In this section (notations being the same as in the Introduction) we describe explicitly the basis elements over the ring $\mathbf{Q}\left[g_{2}, g_{3}\right]$ which generate the first homology with compact supports of the Weierstrass Family over the field of rational numbers $\mathbf{Q}, H_{1}^{c}\left(U, \mathbf{Q}\left[g_{2}, g_{3}\right]\right)$. By the definition of the lifted $p$-adic homology with compact supports [6, p. 415], applied to the characteristic zero case, we have $H_{1}^{c}\left(U, \mathbf{Q}\left[g_{2}, g_{3}\right]\right)$

$=H^{3}\left(\mathbf{A}^{2}\left(\operatorname{Spec}\left(\mathbf{Q}\left[g_{2}, g_{3}\right]\right)\right), \mathbf{A}^{2}\left(\operatorname{Spec}\left(\mathbf{Q}\left[g_{2}, g_{3}\right]\right)\right)-U, \Gamma_{\mathbf{Q}\left[g_{2}, g_{3}\right]}^{*}\left(\operatorname{Spec}\left(\mathbf{Q}\left[g_{2}, g_{3}\right]\right)\right)\right)$.

If one tensors $H_{1}^{c}\left(U, \mathbf{Q}\left[g_{2}, g_{3}\right]\right)$ with $\Delta^{-1} \mathbf{Q}\left[g_{2}, g_{3}\right]$ over $\mathbf{Q}\left[g_{2}, g_{3}\right]$, one has the free $\Delta^{-1} \mathbf{Q}\left[g_{2}, g_{3}\right]$-module of rank two, where $\Delta=g_{2}^{3}-27 g_{3}^{2}$. This is so because we have the universal coefficients spectral sequence

$$
E_{0,1}^{2}=H_{1}^{c}\left(U, \mathbf{Q}\left[g_{2}, g_{3}\right]\right) \otimes_{\mathbf{Q}\left[g_{2}, g_{3}\right]} \Delta^{-1} \mathbf{Q}\left[g_{2}, g_{3}\right] \underset{\sim}{\sim} H_{1}^{c}\left(U, \Delta^{-1} \mathbf{Q}\left[g_{2}, g_{3}\right]\right),
$$

and $\Delta^{-1} \mathbf{Q}\left[g_{2}, g_{3}\right]$ means that the ring $\mathbf{Q}\left[g_{2}, g_{3}\right]$ is localized at the discriminant $\Delta$. The computation has been made even in the $p$-adic case in [1] for this open subfamily of the Weierstrass Family. 
THEOREM 1. Consider $U=\mathbf{W}_{\mathbf{Q}} \cap \mathbf{A}^{2}\left(\operatorname{Spec}\left(\mathbf{Q}\left[g_{2}, g_{3}\right]\right)\right)$, which is the closed affine subscheme of $\mathbf{A}^{2}\left(\operatorname{Spec}\left(\mathbf{Q}\left[g_{2}, g_{3}\right]\right)\right)$. Then the first homology with compact supports $H_{1}^{c}\left(U, \mathbf{Q}\left[g_{2}, g_{3}\right]\right)$ is generated by $\left\{C^{-l} d X \wedge d y\right\}_{l \geqslant 1}$ and $\left\{X C^{-l} d X \wedge d Y\right\}_{l \geqslant 1}$ as a $\mathbf{Q}\left[g_{2}, g_{3}\right]$-module.

REMARK 1. For the pair of affine schemes

$$
\mathbf{A}^{2}\left(\operatorname{Spec}\left(\mathbf{Q}\left[g_{2}, g_{3}\right]\right)\right) \text { and } \mathbf{A}^{2}\left(\operatorname{Spec}\left(\mathbf{Q}\left[g_{2}, g_{3}\right]\right)\right)-U \text {, }
$$

where $U$ is the closed subscheme corresponding to the polynomial $C=Y^{2}-4 X^{3}+$ $g_{2} X+g_{3}$ in $\mathbf{Q}\left[g_{2}, g_{3}, X, Y, Z\right]$, there is induced a long exact sequence of hypercohomology groups,

$$
\begin{aligned}
\cdots \stackrel{\partial^{n-1}}{\rightarrow} H^{n}\left(\mathbf{A}^{2}(A), \mathbf{A}^{2}(A)-U, \Gamma_{A}^{*}\left(\mathbf{A}^{2}(A)\right)\right) \rightarrow H^{n}\left(\mathbf{A}^{2}(A), \Gamma_{A}^{*}\left(\mathbf{A}^{2}(A)\right)\right) \\
\quad \rightarrow H^{n}\left(\mathbf{A}^{2}(A)-U, \Gamma_{A}^{*}\left(\mathbf{A}^{2}(A)\right)\right) \stackrel{\partial^{n}}{\rightarrow} \cdots
\end{aligned}
$$

where $A=\operatorname{Spec}\left(\mathbf{Q}\left[g_{2}, g_{3}\right]\right)$.

There are three first-quadrant spectral sequences induced which have the above three hypercohomology groups as their abutments:

$$
\left\{\begin{array}{l}
{ }^{\prime} E_{1}^{p . q}=H^{q}\left(\mathbf{A}^{2}(A)-U, \Gamma_{A}^{p}\left(\mathbf{A}^{2}(A)\right)\right), \\
E_{1}^{p . q}=H^{q}\left(\mathbf{A}^{2}(A), \Gamma_{A}^{p}\left(\mathbf{A}^{2}(A)\right)\right), \\
{ }^{\prime} E_{1}^{p . q}=H^{q}\left(\mathbf{A}^{2}(A), \mathbf{A}^{2}(A)-U, \Gamma_{A}^{p}\left(\mathbf{A}^{2}(A)\right)\right) .
\end{array}\right.
$$

LEMMA 1. We have the following isomorphisms: the abutment

$$
{ }^{\prime \prime} E^{3}=H^{3}\left(\mathbf{A}^{2}(A), \mathbf{A}^{2}(A)-U, \Gamma_{A}^{*}\left(\mathbf{A}^{2}(A)\right)\right) \underset{\leftarrow}{\approx} E_{2}^{2,1},
$$

and

$$
{ }^{\prime \prime} E^{3} \underset{\leftarrow}{\approx} E^{2}=H^{2}\left(\mathbf{A}^{2}(A)-U, \Gamma_{A}^{*}\left(\mathbf{A}^{2}(A)\right)\right) \stackrel{\approx}{\leftarrow} \operatorname{coker}\left({ }^{\prime} E_{1}^{2,0} \leftarrow^{\prime} E_{1}^{1,0}\right) .
$$

Proof of Lemma 1. Consider the following diagram (Diagram A) with exact rows. We denote the structure sheaf of the affine scheme $\mathbf{A}^{2}(A)=\mathbf{A}^{2}\left(\operatorname{Spec}\left(\mathbf{Q}\left[g_{2}, g_{3}\right]\right)\right)$ by $\Theta_{\mathbf{A}^{2}(A)}$. Therefore, we have " $E^{p, q}=0$ unless $q=1$, which is abutting $" E^{3}=$ $H^{3}\left(\mathbf{A}^{2}(A), \mathbf{A}^{2}(A)-U, \Gamma_{A}^{*}\left(\mathbf{A}^{2}(A)\right)\right)$. Then the isomorphism " $E_{2}^{2,1} \rightarrow{ }^{\prime \prime} E^{3}$ in Lemma 1 follows. Furthermore, this diagram can be rewritten as Diagram B. The remaining two isomorphisms in Lemma 1 are obtained from the well-known lemma in homological algebra, i.e., from Diagram B with the exact rows we have the induced exact sequence

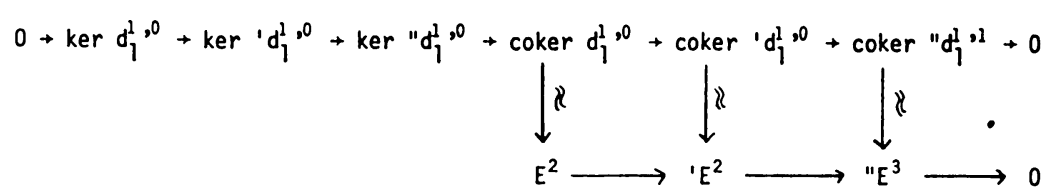




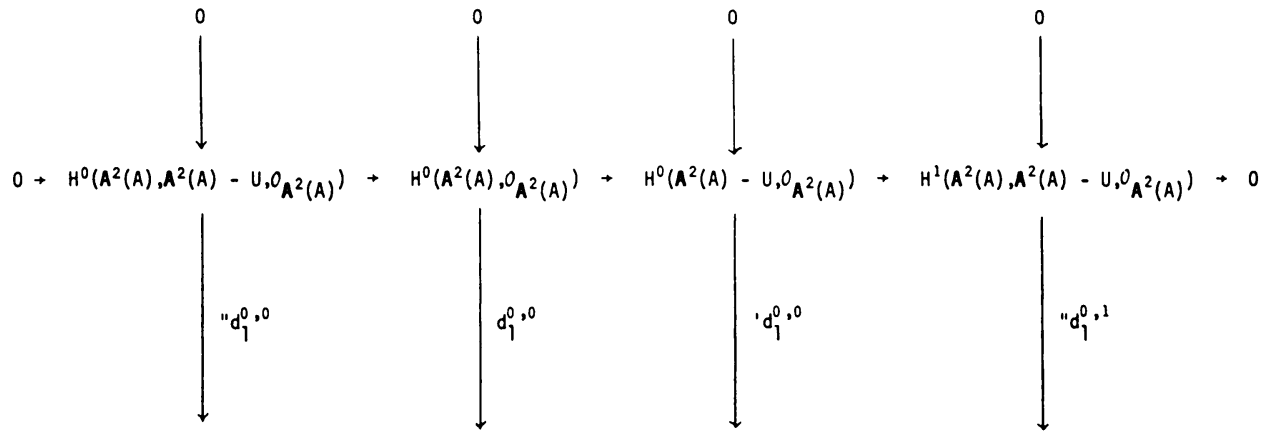

$0+H^{0}\left(A^{2}(A), A^{2}(A)-U, \Gamma_{A}^{1}\left(A^{2}(A)\right)\right)+H^{0}\left(A^{2}(A), \Gamma_{A}^{1}\left(A^{2}(A)\right)\right)+H^{0}\left(A^{2}(A)-U, \Gamma_{A}^{1}\left(A^{2}(A)\right)\right)+H^{1}\left(A^{2}(A), A^{2}(A)-U, \Gamma_{A}^{1}\left(A^{2}(A)\right)\right) \rightarrow 0$

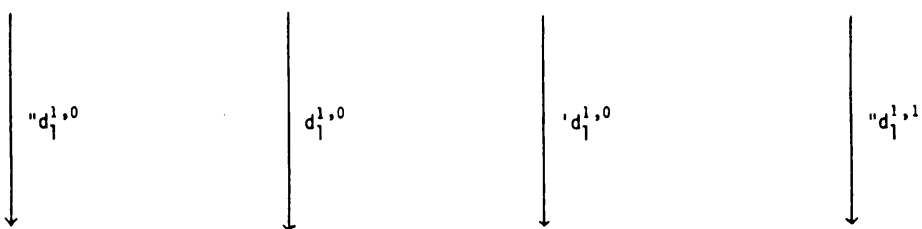

$0 \rightarrow H^{0}\left(\boldsymbol{A}^{2}(A), A^{2}(A)-U, \Gamma_{A}^{2}\left(R^{2}(A)\right)\right)+H^{0}\left(A^{2}(A), \Gamma_{A}^{2}\left(A^{2}(A)\right)\right)+H^{0}\left(A^{2}(A)-U, r_{A}^{2}\left(A^{2}(A)\right)\right) \rightarrow H^{1}\left(A^{2}(A), A^{2}(A)-U, \Gamma_{A}^{2}\left(A^{2}(A)\right)\right)+0$
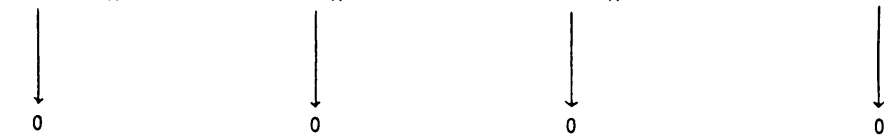

Diagram A

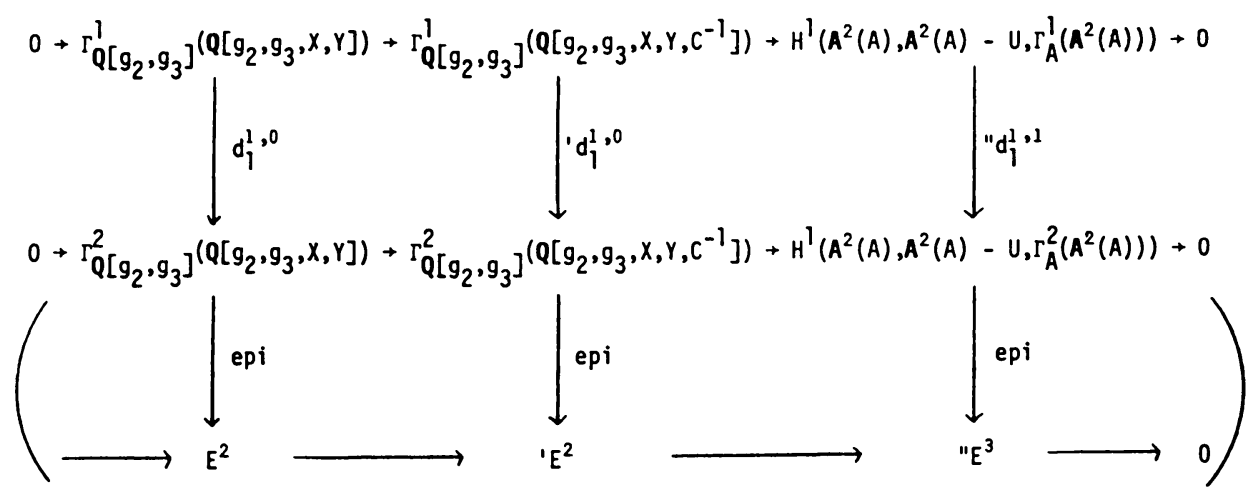

DIAGRAM B

and since the $\mathbf{Q}\left[g_{2}, g_{3}\right]$-homomorphism

$$
d_{1}^{1,0}: E_{1}^{1,0}=\Gamma_{\mathbf{Q}\left[g_{2}, g_{3}\right]}^{1}\left(\mathbf{Q}\left[g_{2}, g_{3}, X, Y\right]\right) \rightarrow E_{1}^{2,0}=\Gamma_{\mathbf{Q}\left[g_{2}, g_{3}\right]}^{2}\left(\mathbf{Q}\left[g_{2}, g_{3}, X, Y\right]\right)
$$

is an epimorphism, we have $E^{2} \approx E_{2}^{2,0} \approx 0$. Therefore

$$
{ }^{\prime} E^{2} \underset{\leftarrow}{\approx} \operatorname{coker}^{\prime} d_{1}^{1,0} \underset{\rightarrow}{\rightarrow} \operatorname{coker}^{\prime \prime} d_{1}^{1,1} \approx{ }^{\prime} E^{3}
$$

as stated in Lemma 1. Q.E.D. 
Hence our computation of the abutment " $E^{3}=H^{3}\left(\mathbf{A}^{2}(A), \mathbf{A}^{2}(A)-U, \Gamma_{A}^{*}\left(\mathbf{A}^{2}(A)\right)\right)$ is reduced to compute

$$
\operatorname{coker}\left(\Gamma_{\mathbf{Q}\left[g_{2}, g_{3}\right]}^{1}\left(\mathbf{Q}\left[g_{2}, g_{3}, X, Y, C^{-1}\right]\right) \stackrel{d_{1}^{1.0}}{\rightarrow} \Gamma_{\mathbf{Q}\left[g_{2}, g_{3}\right]}^{2}\left(\mathbf{Q}\left[g_{2}, g_{3}, X, Y, C^{-1}\right]\right)\right) .
$$

Proof of Theorem 1. From now on we denote, " $d$ ", instead of the exterior differential, " ${ }_{1}^{1,0}$ " in the spectral sequence. We have that

$$
\begin{aligned}
& \text { (1) } d\left(C^{-k} X^{i} Y^{j} d X\right)=\left(-2 k C^{-k-1} X^{i} Y^{j+1}+j C^{-k} X^{i} Y^{j-1}\right) d Y \wedge d X, \\
& \text { (2) } d\left(C^{-k} X^{i} Y^{j} d Y\right) \\
& =\left(12 k C^{-k-1} X^{i+2} Y^{j}-g_{2} k C^{-k-1} X^{i} Y^{j}+i C^{-k} X^{i-1} Y^{j}\right) d X \wedge d Y,
\end{aligned}
$$

in the $\mathbf{Q}\left[g_{2}, g_{3}\right]$-module $\Gamma_{\mathbf{Q}\left[g_{2}, g_{3}\right]}^{2}\left(\mathbf{Q}\left[g_{2}, g_{3}, X, Y, C^{-1}\right]\right)$, where $C=Y^{2}-4 X^{3}+$ $g_{2} X+g_{3}, i, j$ and $k$ are nonnegative integers. The equations (1) and (2) give the cohomologous relations, which are denoted by " ", as

$$
2 k C^{-k-1} X^{i} Y^{j+1} d X \wedge d Y \sim j C^{-k} X^{i} Y^{j} d X \wedge d Y
$$

and

$$
\left(12 k C^{-k-1} X^{i+2} Y^{j}-g_{2} k C^{-k-1} X^{i} Y^{j}+i C^{-k} X^{i-1} Y^{j}\right) d X \wedge d Y \sim 0 .
$$

Notice that, by Lemma 1:

$$
" E_{2}^{2,1} \stackrel{\approx}{\leftarrow} E_{1}^{2,1} / \operatorname{Im}\left(" E_{1}^{1.1} \rightarrow " E_{1}^{2,1}\right)
$$

and

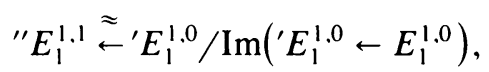

where $E_{1}^{1,0} \approx \Gamma_{\mathbf{Q}\left[g_{2}, g_{3}\right]}^{1}\left(\mathbf{Q}\left[g_{2}, g_{3}, X, Y\right]\right)$. Therefore it suffices to consider the integer $k \geqslant 1$ in the equations (1), (2), (3) and (4) above.

If $j=0$ in (3), then $C^{-k-1} X^{i} Y d X \wedge d Y \sim 0$ for all $i \geqslant 0$ and $k \geqslant 1$. But (4) implies that $C^{-1} X^{i} Y d X \wedge d Y \sim 0$ for $i \geqslant 0$ since $i C^{-1} X^{i-1} Y d X \wedge d Y \sim$ $g_{2} C^{-2} X^{i} Y d X \wedge d Y-12 C^{-2} X^{i+2} Y d X \wedge d Y$. Therefore,

$$
C^{-k} X^{i} Y d X \wedge d Y \sim 0 \text { for all integers } i, k \geqslant 0 .
$$

For any odd integer $j>1$ we have $C^{-k} X^{i} Y^{j} d X \wedge d Y \sim 0$ by combining (3) and (5) and the repeated use of (4). For example, for $j=3$, we have $12 k C^{-k-1} X^{i} Y^{3} d X \wedge$ $d Y \sim 2 C^{-k} X^{i} Y d X \wedge d Y$, which is cohomologous to zero by (5). Then apply (4) for $j=3$ to get

$$
i C^{-k} X^{i-1} Y^{3} d X \wedge d Y \sim g_{3} k C^{-k-1} X^{i} Y^{3} d X \wedge d Y-12 k C^{-k-1} X^{i+3} Y^{3} d X \wedge d Y .
$$

But the right-hand side is cohomologous to zero from the above result. If $i=0$ in (4), we then have

$$
12 k C^{-k-1} X^{2} Y^{j} d X \wedge d Y \sim g_{2} k C^{-k-1} Y^{j} d X \wedge d Y
$$

for all integers $k \geqslant 1$ and $j \geqslant 0$. Especially we have, for $j=0,12 k C^{-k-1} X^{2} d X \wedge d Y$ $\sim g_{2} k C^{-k-1} d X \wedge d Y$. Then it can be plainly seen that

$$
\left(C^{-k} d X \wedge d Y\right)_{k \geqslant 1}, \quad\left(X C^{-k} d X \wedge d Y\right)_{k \geqslant 1} \quad \text { and } \quad\left(X^{i} C^{-1} d X \wedge d Y\right)_{i \geqslant 2}
$$


generate all the elements of the type $X^{i} C^{-k} d X \wedge d Y$ for integers $i \geqslant 0$ and $k \geqslant 0$ over the ring $\mathbf{Q}\left[g_{2}, g_{3}\right]$ from equations (3) and (4). In particular, $X^{2} C^{-1} d X \wedge d Y \sim$ $X^{2} Y^{2} C^{-2} d X \wedge d Y$ by (3) for letting $i=2, j=1$ and $k=1$, but $X^{2} Y^{2} C^{-2} d X \wedge d Y$ $\sim Y^{2} C^{-2} d X \wedge d Y$ by (4) for $i=0, j=2$ and $k=1$; furthermore, $Y^{2} C^{-2} d X \wedge d Y$ is cohomologous to $C^{-1} d X \wedge d Y$ from (3) for $i=0, j=1$ and $k=1$. Hence we have established that $X^{2} C^{-1} d X \wedge d Y \sim C^{-1} d X \wedge d Y$. Next we claim that all the elements of the type $\left(X^{i} C^{-1} d X \wedge d Y\right)_{i \geqslant 3}$ are generated by the two elements $C^{-1} d X \wedge d Y$ and $X C^{-1} d X \wedge d Y$ over the ring $\mathbf{Q}\left[g_{2}, g_{3}\right]$. We have the following recursive formula for integers $i \geqslant 3$ from (3) and (4):

$$
\begin{aligned}
4 X^{i} C^{-1} d X \wedge d Y \sim & g_{2}\left(\frac{1}{12(i-2)}+1\right) X^{i-2} C^{-1} d X \wedge d Y \\
& +\left(g_{3}-\frac{1}{i-2}\right) X^{i-3} C^{-1} d X \wedge d Y
\end{aligned}
$$

Therefore it follows from this recursive formula that $\left(X^{i} C^{-1} d X \wedge d Y\right)_{i \geqslant 3}$ are generated by $C^{-1} d X \wedge d Y$ and $X C^{-1} d X \wedge d Y$ over $\mathbf{Q}\left[g_{2}, g_{3}\right]$. We have established the statement of Theorem 1 for the elements $X^{i} Y^{j} C^{-k} d X \wedge d Y$ with $i \geqslant 1, j=0$ and $k \geqslant 1$. Now we need consider the elements $X^{i} Y^{j} C^{-k} d X \wedge d Y$ for $j=1,2,3, \ldots$ As noted before, we know that if $j$ is an odd integer, $X^{i} Y^{j} C^{-k} d X \wedge d Y \sim 0$. If $j$ is an even integer, the repeated use of (3) and (4) for the elements $X^{i} Y^{j} C^{-1} d X \wedge d Y, i \geqslant 1$ and $j \geqslant 1$, provides the generation of the first homology with compact supports $H_{1}^{c}\left(U, \mathbf{Q}\left[g_{2}, g_{3}\right]\right)$ of the Weierstrass Family by the elements $\left(C^{-k} d X \wedge d Y\right)_{k \geqslant 1}$ and $\left(X C^{-k} d X \wedge d Y\right)_{k \geqslant 1}$. Q.E.D.

Proposition 1. Assumptions and notations being the same as in Theorem 1, $H_{1}^{c}\left(U, \mathbf{Q}\left[g_{2}, g_{3}\right]\right) \otimes_{\mathbf{Q}\left[g_{2}, g_{3}\right]}\left(\Delta^{-1} \mathbf{Q}\left[g_{2}, g_{3}\right]\right)$ is a free $\left(\Delta^{-1} \mathbf{Q}\left[g_{2}, g_{3}\right]\right)$-module of rank two, i.e., it is generated by $X C^{-1} d X \wedge d Y$ and $C^{-1} d X \wedge d Y$, where $\Delta$ is the discriminant, $\Delta=g_{2}^{3}-27 g_{3}^{2}$, and $\Delta^{-1} \mathbf{Q}\left[g_{2}, g_{3}\right]$ is localized at the discriminant $\Delta$.

Proof of Proposition 1. For any integer $i \geqslant 2$ we have

$$
C^{-(i-1)}=C^{-i}\left(Y^{2}-4 X^{3}+g_{2} X+g_{3}\right),
$$

where $d X \wedge d Y$ is omitted for simplicity, and from equations (3), (4) and (6) we have the following cohomologous relation for $i \geqslant 2$ :

$$
\frac{6 i-11}{6(i-1)} C^{-(i-1)} \sim \frac{2 g_{2}}{3} X C^{-i}+g_{3} C^{-i} .
$$

Similarly, one has the corresponding formula for $X C^{-(i-1)}$ by the equations (3), (4) and (6):

$$
\frac{6 i-13}{6(i-1)} X C^{-(i-1)} \sim \frac{g_{2}^{2}}{18} C^{-i}+g_{3} X C^{-i} .
$$


We finally have for $i \geqslant 2$,

(1.3)

$C^{-i} d X \wedge d Y \sim \frac{18}{\Delta}\left\{\frac{g_{2}(6 i-13)}{6(i-1)} X C^{-(i-1)} d X \wedge d Y-\frac{g_{3}(6 i-11)}{4(i-1)} C^{-(i-1)} d X \wedge d Y\right\}$

from equations (1.1) and (1.2).

Equations (1.3) and (1.1) prove that $H_{1}^{c}\left(U, \mathbf{Q}\left[g_{2}, g_{3}\right]\right) \otimes_{\mathbf{Q}\left[g_{2}, g_{3}\right]}\left(\Delta^{-1} \mathbf{Q}\left[g_{2}, g_{3}\right]\right)$ is generated by $X C^{-1} d X \wedge d Y$ and $C^{-1} d X \wedge d Y$ as a $\left(\Delta^{-1} \mathbf{Q}\left[g_{2}, g_{3}\right]\right)$-module. Q.E.D.

COROLlaRY 1. Let $\mathbf{V}_{\mathbf{Q}}^{0}$ be the closed subfamily defined by " $g_{2}=0$ " of the whole Weierstrasss Family $W_{\mathbf{Q}}$. Then the first homology with compact supports,

$$
H_{1}^{c}\left(\mathbf{V}_{\mathbf{Q}}^{0} \cap \mathbf{A}^{2}\left(\operatorname{Spec} \mathbf{Q}\left[g_{3}\right]\right), \mathbf{Q}\left[g_{3}\right]\right)
$$

is generated by $\left\{C^{-k} d X \wedge d Y\right\}_{k \geqslant 1}$ and $\left\{X C^{-k} d X \wedge d Y\right\}_{k \geqslant 1}$ as a $\mathbf{Q}\left[g_{3}\right]$-module.

Proof. In (1.1) and (1.2) in the proof of Proposition 1, we have the following corresponding equations for the closed subfamily $\mathbf{V}_{\mathbf{Q}}^{0}$ defined by " $g_{2}=0$ ":

$$
\begin{aligned}
& \frac{12 i-22}{12(i-1)} C^{-(i-1)} \sim g_{3} C^{-i}, \\
& \frac{6 i-13}{6(i-1)} X C^{-(i-1)} \sim g_{3} X C^{-i} .
\end{aligned}
$$

Then the statement of Corollary 1 follows plainly from $(1.1)^{0}$ and $(1.2)^{0}$. Q.E.D.

Note 1. The equations $(1.1)^{0}$ and $(1.2)^{0}$ also show that Corollaries 2 and 3 are true.

COROLlaRY 2. The first homology with compact supports of the singular fibre $U_{\wp}$ over a point $\wp=\left(g_{2}=0, g_{3}=0\right) \in \operatorname{Spec}\left(\mathbf{Q}\left[g_{2}, g_{3}\right]\right)$, a projective line with a cusp (or $\left.\wp=\left(g_{3}=0\right) \in \operatorname{Spec}\left(\mathbf{Q}\left[g_{3}\right]\right)\right), H_{1}^{c}\left(U_{\wp}, \mathbf{Q}\right)$, is trivial.

COROllary 3. Notations being the same as in Proposition 1,

$$
H_{1}^{c}\left(\mathbf{V}_{\mathbf{Q}}^{0} \cap \mathbf{A}^{2}\left(\operatorname{Spec}\left(\mathbf{Q}\left[g_{3}\right]\right)\right), \mathbf{Q}\left[g_{3}\right]\right) \otimes_{\mathbf{Q}\left[g_{3}\right]}\left(g_{3}^{-1} \mathbf{Q}\left[g_{3}\right]\right)
$$

is generated by the two elements $C^{-1} d X \wedge d Y$ and $X C^{-1} d X \wedge d Y$, where $g_{3}^{-1} \mathbf{Q}\left[g_{3}\right]$ means the localization of the ring $\mathbf{Q}\left[g_{3}\right]$ at $g_{3}$.

REMARK 2. For a point $\wp \neq\left(g_{3}=0\right), H_{1}^{c}\left(U_{\wp}, \mathbf{K}(\wp)\right)$ is generated by $C^{-1} d X \wedge d Y$ and $X C^{-1} d X \wedge d Y$ as a $\mathbf{K}(\wp)$-vector space and where $\mathbf{K}(\wp)$ is the characteristic zero residue field, i.e., $U_{\wp}$ is an elliptic curve. Note that the open subfamily of the Weierstrass Family over $\mathbf{Z} / P \mathbf{Z}$ defined by " $\Delta \neq 0$ " has been computed explicitly using the hypercohomology of a flat lifting with coefficients in the $\dagger$ of sheaves of differential forms, $H^{1}\left(U,\left(\Delta^{-1} \hat{\mathbf{Z}}_{p}\left[g_{2}, g_{3}\right]\right) \dagger \otimes_{\mathbf{Z}} \mathbf{Q}\right)$, where $\left(\Delta^{-1} \hat{\mathbf{Z}}_{p}\left[g_{2}, g_{3}\right]\right) \dagger$ is the $\dagger$ of the localization of the ring $\hat{\mathbf{Z}}_{p}\left[g_{2}, g_{3}\right]$ at the discriminant $\Delta=g_{2}^{3}-27 g_{3}^{2}$, see [1]. The following universal coefficient spectral sequence explains the relationship between Corollary 2 and Theorem 1.

$E_{p, q}^{2}=\operatorname{Tor}_{p}^{\mathbf{Q}\left[g_{2}, g_{3}\right]}\left(H_{q}^{c}\left(U, \mathbf{Q}\left[g_{2}, g_{3}\right]\right), \mathbf{K}(\wp)\right)$ with the abutment $H_{n}^{c}\left(U_{\wp}, \mathbf{K}(\wp)\right)$, where $\wp=\left(g_{2}=g_{3}=0\right) \in \operatorname{Spec}\left(\mathbf{Q}\left[g_{2}, g_{3}\right]\right)$ and $\mathbf{Q}=\mathbf{K}(\wp)$. 
COROLlaRY 4. Let $\mathbf{V}_{\mathbf{Q}}^{3}$ be the closed subfamily of the Weierstrass Family $\mathbf{W}_{\mathbf{Q}}$, defined by " $g_{2}=3$ ". Then $H_{1}^{c}\left(\mathbf{V}_{\mathbf{Q}}^{3} \cap \mathbf{A}^{2}\left(\operatorname{Spec} \mathbf{Q}\left[g_{3}\right]\right), \mathbf{Q}\left[g_{3}\right]\right)$ is generated by $\left\{C^{-k} d X \wedge d Y\right\}_{k \geqslant 1}$ and $\left\{X C^{-k} d X \wedge d Y\right\}_{k \geqslant 1}$ as a $\mathbf{Q}\left[g_{3}\right]$-module. Morover the first homology with compact supports of the singular fibre over the point $\wp=\left(g_{3}=1\right)$ in the base $\operatorname{Spec}\left(\mathbf{Q}\left[g_{3}\right]\right), a$ projective line with an ordinary double point over $\mathbf{K}(\wp)$, is generated by one element as a $\mathbf{K}(\wp)$-vector space. One can then take either $C^{-1} d X \wedge d Y$ or $X C^{-1} d X \wedge d Y$ as the base element for the vector space.

Proof. We only need prove the latter statement. From equations (1.1) and (1.2), we have $(1.1)_{1}^{3}$ and $(1.2)_{1}^{3}$ as follows:

$$
\begin{aligned}
& \frac{6 i-11}{6(i-1)} C^{-(i-1)} \sim 2 X C^{-i}+C^{-i}, \\
& \frac{6 i-13}{6(i-1)} X C^{-(i-1)} \sim \frac{1}{2} C^{-i}+X C^{-i} .
\end{aligned}
$$

Then we have $2(6 i-13) X C^{-(i-1)} \sim(6 i-11) C^{-(i-1)}$ for $i \geqslant 2$. Hence this vector space is one dimensional and the statement of Corollary 4 follows. Q.E.D.

Note 2. For the closed subfamily $\mathbf{V}_{\mathbf{Q}}^{3}$ of the Weierstrass Family we have the following equations $(1.1)^{3},(1.2)^{3}$ and $(1.3)^{3}$ :

$$
\frac{6 i-11}{6(i-1)} C^{-(i-1)} \sim 2 X C^{-i}+g_{3} C^{-i},
$$

$$
\frac{6(i-13)}{6(i-1)} X C^{-(i-1)} \sim \frac{1}{2} C^{-i}+g_{3} X C^{-i},
$$

$(1.3)^{3} \quad\left(g_{3}^{2}-1\right) C^{-i} \sim \frac{1}{6(i-1)}\left\{g_{3}(6 i-11) C^{-(i-1)}-2(6 i-13) X C^{-(i-1)}\right\}$,

for integers $i \geqslant 2$.

Note 3. This paper has been entirely in characteristic zero. The case of nonzero characteristic $p \neq 2,3$ will appear in a forthcoming paper [2], which is a generalization of the paper [1], where an open subfamily " $\Delta \neq 0$ " of the Weierstrass Family was studied.

\section{REFERENCES}

1. G. C. Kato and S. Lubkin, Zeta matrices of elliptic curves, J. Number Theory 15 (1982).

2. G. C. Kato, Lifted p-adic homology with compact supports of Weierstrass family and zeta matrices (in preparation).

3. Bounded Witt vector cohomology of elliptic curves (in preparation).

4. S. Lubkin, Cohomology of completions, Notas de Mat., vol. 42, North-Holland, Amsterdam, 1980.

5. __ A p-adic proof of Weil's conjectures, Ann. of Math. (2) 87 (1968), 105-255.

6. Finite generation of lifted p-adic homology with compact supports. Generalization of the Weil conjectures to singular, noncomplete algebraic varieties, J. Number Theory 11 (1979), 412-464.

Department of Mathematics, California Polytechnic State University, San luis Obispo, CALIFORNIA 93407 